\title{
Conhecimento tradicional de plantas medicinais na comunidade ribeirinha do Igarapé Banha no Município de Mazagão - Amapá, Amazônia brasileira
}

\author{
Traditional knowledge of medicinal plants in the ribeirinha community of \\ Igarapé Banha in the Municipality of Mazagão - amapá, Amazônia brasileira
}

\author{
Mauricio José Cordeiro Souza ${ }^{1}$ \\ Samara Lutyelle Xavier Lobato ${ }^{2}$ \\ Rubens Alex de Oliveira Menezes ${ }^{3}$
}

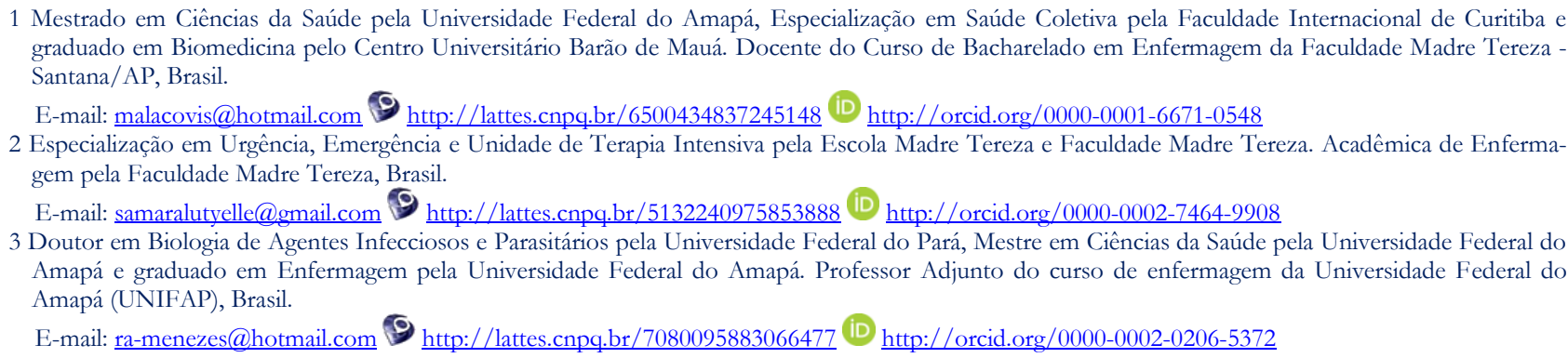

RESUMO: Este estudo objetivou descrever o conhecimento popular de plantas medicinais empregadas pelos moradores ribeirinhos da comunidade do Igarapé Banha, em Mazagão no Amapá, região Amazônica brasileira. Trata-se de um estudo descritivo e exploratório com abordagem quantiqualitativa, elaborado a partir de entrevistas semiestruturada, sendo incluídas 100 pessoas no período de outubro a dezembro de 2017. Do total de entrevistados, $96 \%(96 / 100)$ consideram que possuem conhecimento para usar adequadamente as plantas no tratamento de saúde e $84 \%(84 / 100)$ disseram ter recebido esse conhecimento através de seus pais. Quanto ao uso das plantas para tratamento de saúde $71 \%(71 / 100)$ confirmaram seu uso, $55 \%(55 / 100)$ consideram que as plantas possuem um efeito melhor do que os medicamentos industrializados, $92 \%(92 / 100)$ confia e fica satisfeita com os resultados alcançados e $98 \%$ referiram que não conhecem nenhum efeito, reação ou contraindicação do uso de plantas medicinais. Conclui-se que é notável o uso das plantas medicinais neste estudo, sendo uma herança cultural ao tratamento terapêutico de diversas doenças associado aos conhecimentos empíricos e crenças indígenas e caboclas. A sua utilização trata-se de uma alternativa barata, eficaz e de fácil acesso, além de ser uma prática aceita pelo Sistema Único de Saúde.

Palavras-chave: Etnobotânica. Fitoterapia. Medicamentos. Autocuidado. Saúde. Visita domiciliar.

\begin{abstract}
This study aimed to describe the popular knowledge of medicinal plants used by the residents of the Igarapé Banha community in Mazagão, Amapá, in the Brazilian Amazon region. This is a descriptive and exploratory study with a quantitative-qualitative approach, based on semistructured interviews, with 100 people included in the period from October to December 2017. Of the total number of interviewees, $96 \%(96 / 100)$ knowledge to use plants properly in health treatment, and 84\% (84/100) said they received this knowledge through their parents. As to the use of plants for health treatment, $71 \%(71 / 100)$ confirmed their use, $55 \%(55 / 100)$ consider that plants have a better effect than industrialized medicines, $92 \%(92 / 100)$ trust and is satisfied with the results achieved and 98\% reported that they do not know of any effect, reaction or contraindication to the use of medicinal plants. It is concluded that the use of medicinal plants in this study is remarkable, being a cultural heritage to the therapeutic treatment of several diseases associated to empirical knowledge and indigenous and caboclas beliefs. Its use is a cheap, effective and easily ac-
\end{abstract}


cessible alternative, and is a practice accepted by the Unified Health System.

Keywords: Ethnobotany. Phytotherapy. Medicines. Self-care. Health. Home visit.

\section{INTRODUÇÃO}

O uso curativo das plantas é um recurso tão antigo quanto a espécie humana. O Brasil é um dos países com maior diversidade biológica a partir da presença da floresta amazônica, abrigando desta forma um significativo grupo de populações ribeirinhas, indígenas e tradicionais, que são possuidoras de um amplo conhecimento sobre as plantas existentes em seu ambiente, e seus diversos fins. Esses conhecimentos e práticas são passados de geração em geração, consistindo muitas vezes no único meio de garantia de reabilitação da saúde principalmente para populações que ficam distantes dos centros urbanos (VÁSQUEZ; MENDONÇA; NODA, 2014).

A prática de usos de plantas medicinais para tratamento de saúde é conhecida como fitoterapia, uma prática fortalecida principalmente após a criação Política Nacional de Práticas Integrativas e Complementares (PNPIC) no Sistema Único de Saúde (SUS) pela Portaria $\mathrm{n}^{\circ}$ 971, de 03 de maio de 2006, com objetivo de aumentar as alternativas terapêuticas dos clientes do SUS a partir de fitoterápicos, assegurando qualidade, eficácia e segurança, garantindo acesso as plantas medicinais, bem como a busca pela integralidade da atenção à saúde (BADKE et al., 2012).

Complementando esta política foi instituído o Programa Nacional de Plantas Medicinais e Fitoterápicos (PNPMF), tendo como meta implantar, com segurança, eficácia e qualidade, plantas medicinais, fitoterápicos e serviços relacionados à Fitoterapia no SUS, de forma a desenvolver e assentir as práticas populares e tradicionais de uso de plantas medicinais e remédios caseiros no âmbito do SUS (BRASIL, 2010). Em fevereiro de 2009, o Ministério da Saúde anunciou a Relação Nacional de Plantas Medicinais de Interesse ao
SUS (RENISUS), na qual estão contendo 71 espécies vegetais utilizadas pelas populações e confirmadas cientificamente, e em novembro de 2011, a ANVISA lançou o Formulário de Fitoterápicos da Farmacopeia Brasileira, e dez anos após a implantação da política lança o Memento de Fitoterápicos da Farmacopeia Brasileira (MFFB), um grande avanço para o uso racional e seguro das plantas medicinais

Essa política está sendo implementada no âmbito do SUS por acreditar-se que os cuidados por meio das plantas tragam melhores benefícios a saúde humana, desde que se tenha conhecimento prévio da sua finalidade, de seus riscos e benefícios, sendo importante estudos que buscam fortalecer e resgatar o uso de plantas nestas populações tradicionais e suscitar a reflexão entre os profissionais de saúde quanto a importância de levar em conta esta prática (HARAGUCHI; CARVALHO, 2010). Para Badke et al. (2012), mesmo com o desenvolvimento dos fármacos sintéticos, as plantas medicinais permanecem como alternativas de tratamento, acreditando que o cuidado por este meio seja favorável a saúde, onde a equipe de saúde, em especial o enfermeiro, deve ter um cuidado singular, buscando estimular e resgatar tal recurso de origem popular.

Partindo dessa premissa, este estudo busca conhecer as espécies de plantas medicinais e práticas integrativas em saúde empregadas pelos moradores da comunidade ribeirinha do Igarapé Banha no município de Mazagão, Estado do Amapá, para tratar os problemas de saúde mais corriqueiros da localidade, bem como a participação da equipe de saúde. Considerando que esta comunidade se encontra localizada em uma região de difícil acesso e com baixa cobertura dos serviços prestados pelo Sistema Único de Saúde (SUS), a mesma utiliza plantas medicinais e outras práticas 
terapêuticas integrativas no alívio, tratamento ou cura dos principais problemas de saúde locais, não havendo também nenhuma influência da equipe de saúde na realização das mesmas (BRASIL, 2012; LIMA et al., 2013).

As práticas integrativas e tradicionais de tratamento de saúde com o uso de plantas medicinais já estão integradas no contexto do SUS, e por entender-se que as mesmas trazem benéficos a saúde e contribuem para a integralidade das ações junto a populações residentes em localidades de difícil acesso em vários locais do Brasil, especialmente nos estados amazônicos, onde os serviços de saúde são insuficientes e pouco efetivos e as distâncias são longas e dificultam o acesso principalmente as populações ribeirinhas (LIMA et al., 2013; NASCIMENTO et al., 2013; TEIXEIRA et al., 2014). É relevante que se realizem estudos que possibilitem conhecer essas práticas no âmbito de comunidades tradicionais como a comunidade ribeirinha que se instalou as margens do Igarapé Banha no Município de Mazagão/AP.

O uso de plantas medicinais e práticas tradicionais em regiões amazônicas devem ser incentivados e difundidos, mas sempre de forma correta e orientada para que tenha efetividade e sejam devidamente seguras (NASCIMENTO et al., 2013; TEIXEIRA et al., 2014). O conhecimento das espécies medicinais e as práticas tradicionais em saúde realizadas em comunidades como a do Igarapé Banha é importante para o reconhecimento dessas práticas e o fortalecimento de métodos terapêuticos integrativos locais.

De forma orientada, esse conhecimento contribui como um poderoso aliado da população no auxílio ao tratamento de doenças comuns. Norteados por tais discussões, este estudo teve como objetivo descrever o conhecimento popular de plantas medicinais empregadas pelos moradores ribeirinhos da comunidade do Igarapé Banha, em Mazagão no Amapá, região Amazônica brasileira para alívio, tratamento ou cura dos problemas de saúde local.

\section{ASPECTOS METODOLOGICOS}

\subsection{Tipo de pesquisa}

A presente pesquisa foi de natureza exploratória e descritiva, com abordagem qualiquantitativa. De acordo com Marconi e Lakatos (2010), a pesquisa descritiva tem finalidade de descrever as características ou relações existentes em determinado grupo ou no contexto em que a mesma se realiza, e sua principal contribuição é proporcionar uma nova visão sobre uma realidade já existente.

Para Minayo (2008), a abordagem qualitativa, com aquela em que o cientista objetiva aprofundar-se na compreensão dos fenômenos que estuda, interpretando-os segundo a perspectiva dos próprios sujeitos que participam da situação, sem se preocupar com representatividade numérica, generalizações estatísticas e relações lineares de causa e efeito, enquanto que os métodos quantitativos têm o objetivo de mostrar dados, indicadores e tendências observáveis, ou produzir modelos teóricos abstratos com elevada aplicabilidade prática evidenciado a regularidade dos fenômenos.

Segundo Minayo (2008) a metodologia quali-quantitativa é aquela que envolve métodos qualitativos e quantitativos para a obtenção de uma análise mais profunda do assunto da pesquisa. Esse tipo de pesquisa permite um cruzamento de dados maior, potencializando a validação das informações dos sujeitos da pesquisa.

\subsection{Cenário da pesquisa}

A pesquisa foi realizada na área ribeirinha do município de Mazagão - AP, especificamente na comunidade do Igarapé Banha, localizada a 45 minutos de Santana. O Igarapé 
Banha foi escolhido para realização da pesquisa pela tradição no uso de plantas medicinais de seus moradores e por sua localização, que apesar de próximo as sedes municipais de Mazagão e Santana, devido ao acesso exclusivo por via fluvial e em pequenas embarcações lhe confere certo grau de isolamento.

Vivem nesta comunidade, segundo o cadastro do programa de agentes comunitários de saúde 873 pessoas distribuídas em 82 famílias. A economia local baseia-se na agricultura familiar de subsistência (plantio de banana, mandioca, abóbora, milho, árvores frutíferas, açaí, etc.), na pesca artesanal, no transporte fluvial de moradores e visitantes e também de incentivos sociais como bolsa família e seguro defeso (auxílio financeiro temporário às famílias de pescadores na época de procriação de peixes - piracema).

Não há serviço de saúde disponível, apenas uma agente comunitária de saúde faz o acompanhamento dessas famílias e quando se faz necessário, algum tipo de atendimento à saúde, os moradores procuram o município de Santana para o atendimento de suas necessidades. É em Santana também que fazem aquisição de produtos, que não são produzidos na comunidade, alimentos, higiene, e limpeza, e que comercializam o excedente de sua produção de subsistência, adquirindo os recursos necessários para suas famílias, embora a comunidade pertença ao município de Mazagão. As moradias, quase na sua totalidade, são construções tipo palafitas às margens do Igarapé e são desprovidas de água tratada e rede de esgoto.

\subsection{Sujeitos da pesquisa}

A seleção dos sujeitos da pesquisa foi feita através de visitas domiciliares prévias buscando identificar pessoas acima de 18 anos que demonstraram conhecimento sobre o uso de plantas medicinais e práticas de saúde alternativas dentro da comunidade, buscando atingir uma amostra de $10 \%$ da população, resultando em aproximadamente 87 pessoas. Foram entrevistadas 100 pessoas, no período de outubro a dezembro de 2017, sendo realizadas três visitas nas casas dos moradores do povoado. $\mathrm{Na}$ primeira identificando os moradores que se adequavam aos critérios de inclusão, na segunda foram coletados os dados utilizando a técnica da entrevista semiestruturada e uma terceira visita ocorreu para realização de uma palestra informativa, com distribuição de mudas e cartilha sobre a utilização das plantas medicinais pelos usuários da comunidade.

\subsection{Critérios de inclusão e exclu- são}

Fizeram parte da pesquisa indivíduos entre 18 a 75 anos de ambos os sexos, moradores permanentes da comunidade do Igarapé Banha, com conhecimento sobre o uso de plantas medicinais, que estevam na comunidade no período da coleta de dados, e aceitaram participar do estudo.

Como critério de exclusão não morar na comunidade, estar ausente da comunidade no período da pesquisa, ser menor de 18 anos, apresentar distúrbios cognitivos e/ou de comunicação e negar e/ou desistir de colaborar com o estudo. Vale ressaltar que nenhum dos selecionados se recusou ou desistiu de participar do estudo.

\subsection{Coleta de dados}

Os dados foram coletados através de entrevista semiestruturada, que se caracteriza por uma conversa com finalidade específica guiada por um roteiro de perguntas aberta e fechadas conforme objetivos propostos, dando ao entrevistado a possibilidade de falar mais livremente sobre o tema proposto (MINAYO, 2008).

As entrevistas foram realizadas pelos pes- 
quisadores, conforme a disponibilidade do entrevistado em local e hora determinado pelo mesmo, individualmente, e nas residências do entrevistado. Foram gravadas e transcritas de modo a permitir a organização do material por palavras, temas, tópicos e categorias analíticas.

Foram realizadas três visitas a comunidade. $\mathrm{Na}$ primeira visita o objetivo foi identificar os moradores que se adequavam aos critérios de inclusão, principalmente na questão de ter conhecimento sobre as plantas medicinais. Não houve entrevista, apenas conversação com alguns moradores (possíveis lideranças na comunidade), tais como líderes religiosos, agentes de saúde, moradores antigos entre outros, além da observação por parte dos pesquisadores dos hábitos e costumes locais.

$\mathrm{Na}$ segunda visita foram coletados os dados utilizando a técnica da entrevista semiestruturada, nas casas dos sujeitos. Foi totalizado um quantitativo de 100 (cem) entrevistas realizadas, visitadas $100 \%$ das residências da comunidade, e entrevistado pelo menos um sujeito em cada, sendo que em algumas mais de um sujeito. Nesta segunda visita encerrouse a coleta de dados, por meio de entrevistas, que foram utilizados para análise e conclusões do estudo.

Uma terceira visita ocorreu para realização de uma palestra e distribuição de cartilha sobre plantas medicinais e distribuição de mudas, em dia e local previamente agendado através de convite a comunidade. Esta última visita teve como objetivo principal incentivar o cultivo e resgatar a tradição do uso de plantas nesta comunidade, além de proporcionar aos pesquisadores mais uma oportunidade de conhecer melhor a comunidade e assim poder inferir com mais precisão as análises do conteúdo representado na fala dos sujeitos.

\subsection{Análises dos dados}

Os dados coletados foram analisados quali- tativamente através da técnica de análise de conteúdo, temática que, para Bardin (2009), deve ter como ponto de partida uma organização dos dados coletados possibilitando a execução das fases da análise que são: a préanálise, a exploração do material, tratamento dos resultados e a inferência ou interpretação através da categorização da representatividade das falas dos sujeitos. Para análise quantitativa os dados foram tabulados segundo as categorias tais como: espécies de plantas, uso e indicações, práticas associadas, interesse e aceitação, de forma a identificar a frequência de ocorrência do fenômeno e descrever as principais características da comunidade quanto ao uso de plantas medicinais.

Os dados obtidos foram armazenados em um programa de banco de dados da Microsoft Office Access 2016 e analisados utilizando o software BioEstat 5.0. Os valores foram avaliados pela estatística descritiva, sendo utilizado as frequências absolutas e relativas (\%) das variáveis de maior relevância para a construção da tabela. Para avaliar a relação entre os dados obtidos, foi realizado o teste nãoparamétrico para duas amostras independentes (Teste G), sendo considerados significativos os valores de $p \leq 0,05$.

\subsection{Aspectos éticos}

Este estudo foi desenvolvido observando os preceitos éticos da Resolução 466/2012, certificado pelo Comitê de Ética em Pesquisa da Associação Educacional da Amazônia Faculdade SEAMA - CEP/SEAMA, em 26 de setembro de 2017, protocolo n ${ }^{\circ} 58475316$. 4.0000.5021, em conformidade com os Princípios Éticos em Experimentação Humana, adotados pelo Comitê Nacional de Ética em Pesquisa. Os sujeitos da pesquisa foram convidados a participar da assinatura do Termo de Consentimento Livre e Esclarecido, bem como ao esclarecimento do estudo a respeito da finalidade da pesquisa, dos riscos e benefí- 
cios previstos, o anonimato dos sujeitos e a garantia de que os dados coletados nesta pesquisa foram utilizados apenas para os fins da mesma.

\section{RESULTADOS E DISCUSSÃO}

A pesquisa foi realizada na área ribeirinha do município de Mazagão, especificamente na comunidade do Igarapé Banha, localizada a 45 minutos de Mazagão, Município do estado do Amapá. O Igarapé Banha foi escolhido para realização da pesquisa pela tradição no uso de plantas medicinais pelos seus moradores e por sua localização, encontrando-se relativamente isolado, cujo acesso é exclusivo por via fluvial por pequenas embarcações.

$\mathrm{Na}$ Tabela 1 estão demonstradas as informações sócio demográficas dos entrevistados, tais como, faixa etária, sexo, grau de instrução e ocupação, buscando semelhanças sociais e demográficas que elucidem os objetivos deste estudo. A faixa etária dos entrevistados variou entre 18 a 75 anos, sendo que de 18 a 30 anos e 31 a 50 anos foram as mais prevalentes com $39 \%$ (39/100) cada faixa etária. Segundo Firmo et al (2011), não há um consenso entre estudiosos sobre uma faixa etária de início com que as pessoas mais usam plantas medicinais, mas o que se percebe é a utilização de plantas medicinais por pessoas de idade adulta avançada (ARNOUS; SANTOS; BEINNER, 2005).
Tabela 1 - Perfil dos entrevistados na Comunidade Igarapé Banha - Mazagão - AP, 2016

Table 1 - Profile of respondents in the Igarapé Banha Community - Mazagão - AP, 2016

\begin{tabular}{|c|c|c|c|c|c|c|}
\hline \multirow{2}{*}{ Variáveis } & \multicolumn{2}{|c|}{$\begin{array}{l}\text { Femini- } \\
\text { no }\end{array}$} & \multicolumn{2}{|c|}{$\begin{array}{l}\text { Masculi- } \\
\text { no }\end{array}$} & \multirow{2}{*}{$\begin{array}{c}\text { To- } \\
\text { tal }\end{array}$} & \multirow{2}{*}{$\begin{array}{l}\text { P- } \\
\text { valor* }^{*}\end{array}$} \\
\hline & $(\mathrm{n})$ & $(\%)$ & (n) & $(\%)$ & & \\
\hline Faixa etária & & & & & & 0.7408 \\
\hline $18-30$ & 19 & 48,8 & 20 & 51,2 & 39 & \\
\hline $31-50$ & 15 & 38,4 & 24 & 61,6 & 39 & \\
\hline $51-70$ & 7 & 35 & 13 & 65 & 20 & \\
\hline Acima de 71 & 1 & 50 & 1 & 50 & 2 & \\
\hline Grau de instrução & & & & & & 0.1543 \\
\hline Não alfabetizado & 1 & 100 & 0 & 0 & 1 & \\
\hline $\begin{array}{l}\text { Ensino fundamental Incom- } \\
\text { pleto }\end{array}$ & 23 & 33,9 & 45 & 66,1 & 68 & \\
\hline $\begin{array}{l}\text { Ensino fundamental Comple- } \\
\text { to }\end{array}$ & 4 & 40 & 6 & 60 & 10 & \\
\hline Ensino médio Incompleto & 4 & 57,1 & 3 & 42,9 & 7 & \\
\hline Ensino médio Completo & 9 & 69,2 & 4 & 30,8 & 13 & \\
\hline Ensino superior & 1 & 100 & 0 & 0 & 1 & \\
\hline Ocupação & & & & & & $\begin{array}{l}<0.000 \\
1\end{array}$ \\
\hline Agricultor & 1 & 12,5 & 7 & 87,5 & 8 & \\
\hline Apanhador de açai & 3 & 15 & 17 & 85 & 20 & \\
\hline Aposentado & 3 & 33,3 & 6 & 66,7 & 9 & \\
\hline $\begin{array}{l}\text { Barquei- } \\
\text { ro/catraeiro/pescador }\end{array}$ & 4 & 25 & 12 & 75 & 16 & \\
\hline Dona de casa & 18 & 100 & 0 & 0 & 18 & \\
\hline Estudante & 8 & 61,6 & 5 & 38,4 & 13 & \\
\hline Outras & 5 & 31,2 & 11 & 68,8 & 16 & \\
\hline
\end{tabular}

Nos estudos de Monteles e Pinheiro (2007) e Veiga Junior (2008), foi observado uma prevalência significante do gênero feminino sobre o masculino. O alto percentual de mulheres é condizente com o conhecimento obtido em estudos etnofarmacológicos anteriores de que são as mulheres, que normalmente preparam os alimentos, que também preparam os chás e as infusões à base de plantas (HOWARD, 2003).

Entretanto, essa pesquisa evidenciou em relação ao gênero, dados opostos aos autores supracitados, sendo 58\% (58/100) para o sexo masculino e $42 \%(42 / 100)$ para o feminino. Essa pesquisa não observou o predomínio do gênero feminino nesta comunidade não apresentando diferença estatística (Teste $\mathrm{G}=$ 0.7408) de gênero quanto ao conhecimento e uso de plantas medicinais.

Adicionalmente, quanto a escolaridade prevaleceu com 68\% (68/100) para ambos os sexos para o ensino fundamental incompleto. Apesar de um dado importante, não foi ob- 
servado significância estatística (Teste $G=$ 0.1543). Com relação a ocupação exercida pela população da comunidade, foi observado uma diferença entre os gêneros, aliando a ocupação e a dinâmica cultural da comunidade. Dentre as diversas ocupações relatadas, para as mulheres a ocupação de dona de casa foi a mais prevalente $18 \%(18 / 100)$ e para os homens a ocupação de apanhador de açaí $20 \%$ (20/100), ocupações típicas e comuns a populações rurais. É importante destacar que a variável ocupação foi observada significância estatística (Teste $G<0.0001$ ).

A baixa escolaridade e a baixa remuneração são apresentadas como um fator que influencia e direciona a população ao uso de plantas medicinais. A prática da fitoterapia como alternativa na medicina popular é bem valorizada nas comunidades rurais e populações carentes (ALVES et al., 2015), constituindo-se como uma solução mais acessível para os seus problemas de saúde (SILVA et al., 2011) e muitas vezes se configura como a única alternativa de tratamento (ALVIM et al., 2006).

A fitoterapia é considerada uma prática da medicina tradicional, entretanto, é de extrema importância a correta identificação e classificação botânica das plantas e seus princípios ativos para prevenir o erro (FRANÇA et al., 2008). Na comunidade Igarapé Banha em estudo 96\% (96/100) dos entrevistados consideram que possuem conhecimento para usar adequadamente as plantas no tratamento de saúde e $84 \%$ (84/100) disseram ter recebido esse conhecimento através de seus pais $60 \%$ (60/100), avós $24 \%(24 / 100)$ ou outro familiar 6\% (6/100) (Figura 1).
Figura 1 - Informação sobre a transmissão de conhecimentos quanto ao uso de plantas medicinais - Comunidade Igarapé Banha - Mazagão - AP, 2016.

Figure 1 - Information on the transmission of knowledge regarding the use of medicinal plants - Igarapé Banha Community Mazagão - AP, 2016.

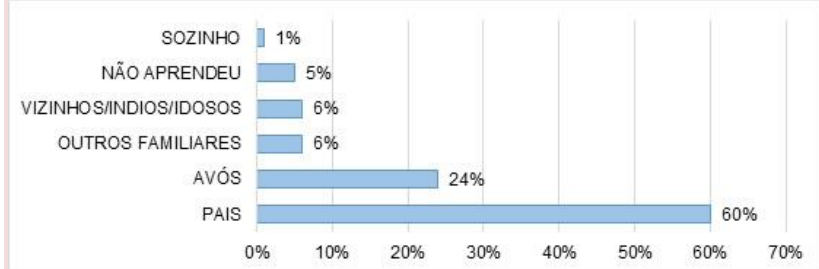

Fonte: Instrumento de Coleta de Dados da Pesquisa

No conteúdo das falas percebe-se a influência familiar na transmissão dos conhecimentos assim como também a referência a população mais antiga.

Aprendi com minha vó ela sabia de muitas plantas (RRS).

Aprendi com os mais antigos da família que ensinavam pra gente fazer pros nossos filhos

(SCL).

Conheci o efeito dos matos com minha sogra (JSM).

O consumo de plantas medicinais tem base na tradição familiar, no fácil acesso às plantas e na possibilidade de preparo caseiro, além do baixo custo. Em geral é resultado da experiência acumulada durante anos do uso e do próprio cultivo dessas plantas medicinais (ALVES et al., 2015). Para Mata (2009), a transmissão oral desses conhecimentos ocorre de geração em geração.

Quanto ao uso das plantas para tratamento de saúde $71 \%(71 / 100)$ responderam que sim, $23 \%(23 / 100)$ as vezes e $6 \%$ não $(6 / 100)$ (figura 2), caracterizando a alta frequência de uso deste recurso na comunidade o que também pode ser percebido nas falas.

Sim eu uso todo tempo que tô com algum problema de saúde (ICR).

Sim a gente conhece muitos tipos de mato (CCC). 
Figura 2 - Informação sobre utilizar plantas medicinais como forma de tratamento e prevenção em saúde - Comunidade Igarapé Banha - Mazagão - AP, 2016.

Figure 2 - Information on using medicinal plants as a form of health treatment and prevention - Igarapé Banha Community Mazagão - AP, 2016

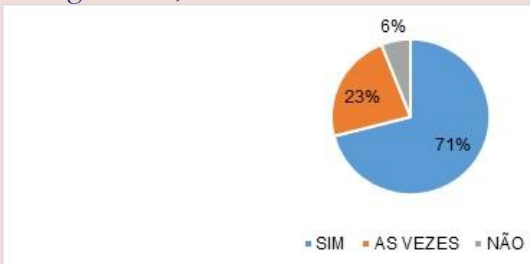

Fonte: Instrumento de Coleta de Dados da Pesquisa

Segundo Brasileiro et al (2008), atualmente alguns fatores têm contribuído para o aumento da utilização deste recurso, entre eles, o alto custo dos medicamentos industrializados, o difícil acesso da população à assistência médica, bem como a tendência, ao uso de produtos de origem natural. Para muitas pessoas que não têm acesso aos serviços de saúde, as plantas medicinais são as principais opções, para o tratamento dos problemas de saúde (FIRMO et al., 2011).

Para Silva et al (2011), o que determina o efeito de uma planta é o contexto no qual ela é usada, seu preparo e dosagem e a concepção de saúde-doença de uma determinada cultura. $\mathrm{Na}$ comunidade do Igarapé Banha 55\% (55/100) dos sujeitos da pesquisa consideram que as plantas possuem um efeito melhor do que os medicamentos industrializados, 36\% (36/100) acham que é igual e 9\% (9/100) acham que as plantas não são eficazes (figura 3).

Figura 3 - Informação sobre a percepção dos entrevistados quanto a comparação do efeito das plantas medicinais em relação aos medicamentos industrializados

Figure 3 - Information on respondents' perception of comparing the effect of medicinal plants on industrialized medicines

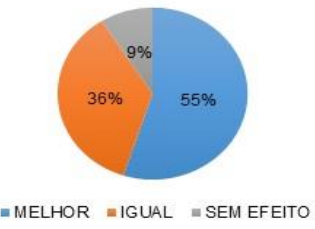

Fonte: Instrumento de Coleta de Dados da Pesquisa

Fica claro então que a comunidade em es- tudo confia plenamente no uso de plantas medicinais para o tratamento de sua saúde. Percebe-se no significado das falas a alta confiabilidade e a segurança que a comunidade deposita neste tipo de terapia.

Ah, o remédio feito das plantas é mais natural, a gente sabe de onde vem (MDC).

Os remédios de farmácia são mais fortes, acho melhor o natural (LERS).

Quanto mais se utiliza plantas mais se percebe que são eficazes, o uso de plantas medicinais vem crescendo junto a população em geral e mais de $2 / 3$ da população do planeta usa plantas medicinais como recurso terapêutico (ARGENTA et al., 2011). Em relação a confiabilidade no tratamento pode-se perceber que a comunidade confia e fica satisfeita com os resultados alcançados, 92\% (92/100) dos entrevistados afirmaram que o tratamento realizado foi um sucesso subtendido então que houve melhora da saúde ou a cura, o que ficou bem explícito na fala dos sujeitos.

Todas as pessoas falam que faz efeito né, ficam satisfeitas (JFS).

Tudo deram positivo até hoje (MSS).

Toda vez que tomei me dei bem, melhorei da dor $(A B C)$.

Há referências na literatura de que a satisfação com uso de plantas medicinais para tratamento de saúde está diretamente relacionada a frequência de uso, quanto mais se usa mais se percebe os efeitos benéficos (RAMOS et al., 2016). É neste contexto que devem ser fomentados estudos que comprovem o efeito e validem as formas de uso, para que as plantas medicinais sejam usadas com mais segurança.

É comum entre as populações acreditar que as plantas são completamente inofensivas, mais seguras que os medicamentos convencionais, que não apresentavam contraindicação e nenhum tipo de reação adversa (SCHIAVO et al., 2014). Acreditam mesmo que por ser 
natural as plantas não vão causar mal à saúde. (FRANÇA et al., 2008). Entretanto muitas plantas medicinais podem ser tóxicas e a diferença entre um remédio e um veneno pode estar na quantidade que é utilizada, pois existem plantas que, mesmo sendo conhecidas como medicinais, são tóxicas (ORGANIZAÇÃO SOCIAL DE SAÚDE VIVA RIO, 2015). Neste estudo $98 \%$ dos sujeitos referiram que não conhecem nenhum efeito, reação ou contraindicação do uso de plantas medicinais. Adicionalmente, algumas falas demonstram a pouca ou nenhuma preocupação com estes possíveis efeitos.

Olha nunca teve quando usei (FMD).

Sempre que usei nunca fez mal (BRA).

Esse contexto, enfatiza e demostra a opinião da comunidade sobre o uso de plantas medicinais. Adicionalmente, o uso terapêutico das plantas medicinais na saúde humana foi construído na sabedoria do senso comum que articula cultura e saúde, num contexto histórico determinado, fruto de um conhecimento acumulado e várias vezes testado e atualizado na prática (ALVIM et al., 2006; MATA, 2009).

Entretanto a facilidade de acesso à medicina moderna e a saída das pessoas de seus ambientes naturais para regiões urbanas, ameaçou essa prática e contribuiu para a perda do conhecimento popular herdado e transferido há várias gerações (ALVES et al., 2015). O conhecimento e as terapêuticas anteriormente empregados na saúde humana, práticas de origem popular, foram marginalizados por não ter base científica (ALVIM et al., 2006).

Contudo o crescimento populacional urbano e as dificuldades dos sistemas de saúde em atender as demandas de saúde da população, o alto custo dos medicamentos industrializados, mas também a busca por uma vida saudável, tem contribuído para que a população retorne a prática de uso de plantas medicinais (SILVEIRA; BANDEIRA; ARRAIS, 2008).

Veiga Junior, Pinto e Maciel (2005) relatam que as plantas medicinais passaram a ser uma ferramenta importante para os profissionais de saúde, usuários, pesquisadores e gestores do SUS e que o conhecimento das atividades farmacológicas e a toxicidade das plantas medicinais de cada bioma brasileiro de acordo com os costumes, tradições e condição socioeconômica da população, deve ser buscado pelos profissionais de saúde.

$\mathrm{Na}$ busca de uma assistência eficaz, abrangente, humanizada e independente da tecnologia farmacêutica, alguns profissionais têm despertado o interesse para estas práticas integrativa, e o enfermeiro tem sido peça chave para adoção e implantação do uso racional e orientado das plantas medicinais unindo o avanço tecnológico ao conhecimento popular, principalmente os profissionais vinculados a Equipe Saúde da Família (ESF), uma vez que criam maiores vínculos com a comunidade, e promovem maior acessibilidade ao atendimento em saúde (SAMPAIO et al., 2013).

É papel dos profissionais de saúde orientar as pessoas quanto ao uso indiscriminado de algumas plantas medicinais, aos riscos das associações de medicamentos, e as possíveis reações adversas, proporcionando a população respaldo na tradição cultural e no conhecimento científico para utilizar racionalmente os fitoterápicos (FRANÇA et al., 2008).

É comum em parte da população principalmente entre as populações onde a tradição do uso de plantas já deu lugar a medicina moderna acreditar que as plantas são completamente inofensivas, mais seguras que os medicamentos convencionais, que não apresentam contraindicação e nenhum tipo de reação adversa (SCHIAVO et al., 2014). A Amazônia tem uma importância relevante quanto a riqueza do conhecimento popular acerca do uso terapêutico de plantas (PASTORE, 2011).

A promoção da saúde por meio de terapias naturais envolve o resgate de valores culturais, ao mesmo tempo em que estimula ações intersetoriais, facilitando o vínculo equipe de 
saúde-comunidade, proporcionando aproximação entre profissionais e usuários, estimulando o cuidado autônomo, o desenvolvimento local, a intersetorialidade e a participação comunitária (SANTOS et al., 2011). O Amapá tem utilizando como base o conhecimento tradicional das comunidades locais do Estado, transformando através de tecnologias próprias, os seus recursos naturais em especial as plantas medicinais, como alternativas de tratamento em saúde.

\section{CONSIDERAÇÕES FINAIS}

$\mathrm{Na}$ comunidade ribeirinha do Igarapé $\mathrm{Ba}$ nha, muitas plantas são utilizadas tradicionalmente pela população para recuperação da saúde, e está prática é repassada de geração em geração, através de informações oral, em geral é um conhecimento familiar bastante difundido na população que tem sido repassado de geração em geração. Eles confiam e acreditam na eficácia deste tipo de tratamento até com um certo exagerado excesso acreditando que as plantas são inofensivas a saúde, não identificando nenhum efeito ou evento adverso nem contraindicações.

Considerando que esta é uma população rural e de baixa escolaridade, baixa renda, e de difícil acesso, essa prática é fortalecida muitas vezes como a única forma de tratamento a saúde acessível aos moradores e é neste contexto que se enfatiza a necessidade de incentivar, resgatar e garantir a continuidade do uso de plantas medicinais na comunidade, através de estudos e projetos que auxiliem no cultivo, preparo e no uso seguro e correto destes recursos.

\section{REFERÊNCIAS}

ALVES, J. J. P. et al. Conhecimento Popular Sobre Plantas Medicinais e o Cuidado da Saúde Primária: Um Estudo de Caso da Comunidade Rural de Mendes, São José de Mipi-
bu/RN. CARPE DIEM: Revista Cultural e Científica do UNIFACEX, v. 13, n. 1, p. 136-156, 2015.

ALVIM, N. A. T. et al. O uso de plantas medicinais como recurso terapêutico: das influências da formação profissional às implicações éticas e legais de sua aplicabilidade como extensão da prática de cuidar realizada pela enfermeira. Revista Latino-americana de enfermagem, v. 14, n. 3, p. 316-323, 2006.

ARGENTA, S. C. et al. Plantas Medicinais: cultura popular versus ciência. Revista eletrônica Vivências, v. 7, n. 12, p. 51-60, 2011. ARNOUS, A.H.; SANTOS, A.S.; BEINNER, R.P.C. Plantas medicinais de uso caseiroconhecimento popular e interesse por cultivo comunitário. Revista Espaço para a Saúde, v.6, n.2, p.1-6. Londrina, 2005.

BADKE, M. R. et al. Saberes e práticas populares de cuidado em saúde com o uso de plantas medicinais. Texto contexto - enfermagem, v. 21, n. 2, p. 363-70, Florianópolis, 2012. https://doi.org/10.1590/S0104-07072012000 200014

BARDIN, L. Análise de conteúdo. Lisboa: Edições 70, 2009. Disponível em: $<$ https://pt.slideshare.net/alasiasantos/analis e-de-conteudo-laurence-bardin $>$. Acesso em 20 jul. 2015.

BRASIL. Conselho Nacional de Saúde. Esclarecimento do Conselho Nacional de Saúde à População Brasileira frente às recentes e negativas reportagens veiculadas na mídia sobre plantas medicinais e fitoterapia. Brasília, 08 de outubro de 2010. Disponível em: <htt p://www.conselho.sau de.gov.br/ultimas_noticias/2010/08_out_pla ntas_medicinais.htm $>$ Acesso em 20 jul. 2017. BRASIL. Ministério da Saúde (MS). Secretaria de Atenção à Saúde. Departamento de Atenção Básica. Práticas integrativas e complementares: plantas medicinais e fitoterapia na Atenção Básica. Brasília: MS; 2012. Disponível em: <http://189.28.128.100/dab/docs/publicaco 
es/geral/miolo_CAP_31.pdf> Acesso em 30 jul. 2017.

BRASILEIRO, B. G. et al. Plantas medicinais utilizadas pela população atendida no "Programa de Saúde da Família", Governador Valadares, MG, Brasil. Revista Brasileira de Ciências Farmacêuticas, v. 44 , n. 4 , p. 629 636, 2008. https://doi.org/10.1590/\$1516-9332200 8000400009

FIRMO, W. C. A. et al. Contexto histórico, uso popular e concepção científica sobre plantas medicinais. Cadernos de Pesquisa, São Luís, v. 18, n. especial, dez. 2011.

FRANÇA, I. S. X. et al. Medicina popular: benefícios e malefícios das plantas medicinais. Revista Brasileira de Enfermagem, v. 61, n. 2, p. 201-208, 2008. https://doi.org/10.1590/ $\underline{\text { S0034-71672008000200009 }}$

HARAGUCHI, L. M. M.; CARVALHO, O. B. (Coord). Plantas Medicinais: do curso de plantas medicinais. São Paulo: Secretaria Municipal do Verde e do Meio Ambiente. Divisão Técnica Escola Municipal de Jardinagem, 2010. Disponível em: <http://www.pre feitura.sp.gov.br/cidade/secretarias/upload/ meio_ambiente/arquivos/plantas_med_web. pdf >. Acesso em: 21 out. 2016.

HOWARD, P.L. Women \& Plants. Gender Relations in Biodiversity Management \& Conservation. Londres: Zed Books Ltd. 2003.

LIMA, R. B. et al. Caracterização agroecológica e socioeconômica dos moradores da comunidade quilombola do Curiaú, Macapá-AP, Brasil. Biota Amazônia, v. 3, n. 3, p. 113138, 2013. https://doi.org/10.18561/2179-5746/ biotaamazonia.v3n3p113-138

MARCONI, M. de A.; LAKATOS, E.M. Metodologia científica. São Paulo: Atlas, 2010.

MATA, N. D. S. A participação da mulher Wajãpi no uso tradicional de plantas medicinais. 2009. 141f. Dissertação (Mestrado Integrado em Desenvolvimento Regional) Universidade Federal do Amapá. UNIFAP, Macapá, 2009.

MINAYO, M. C. S. O desafio do conheci- mento. 11 ed. São Paulo: Hucitec, 2008 MONTELES, R.; PINHEIRO, B.U.C. Plantas medicinais em um quilombo maranhense: Uma perspectiva etnobotânica. Revista de Biologia e Ciência da Terra. v. 7, n. 2, p. 17-37, 2007.

NASCIMENTO, W.M.C.; et al. Plantas medicinais e sua utilização pelas comunidades do município de Sobral, Ceará. SANARE, Sobral, v.12, n.1, p. 46-53, jan./jun. - 2013 PASTORE, J.R. F. Apresentação In: RIOS, M. N. S; PASTORE JR, F.(Org.). Plantas da Amazônia: 450 espécies de uso geral. Universidade de Brasília: Brasília, DF. 2011.

RAMOS, A. S. et al. Conhecimento e uso de plantas medicinais no tratamento de doenças pelos moradores atendidos na unidade básica de saúde da ilha de Santana/AP. Madre Ciência Saúde, v. 1, n. 1, 2016.

ORGANIZAÇÃO SOCIAL DE SAÚDE VIVA RIO, Secretaria Municipal de Saúde. Que tal um chazinho? Guia prático de plantas medicinais. Gerencia do Programa de Práticas Integrativas e Complementares. Rio de Janeiro: SMS, 2015 (Série E. Comunicação e Educação em Saúde). 40 p., il. Disponível em: <http://www.rio.ri.gov.br/docu ments/73801/14163dd4-f62d-4f52-8b0881ff3f625212> Acesso em: 25 mai. 2017. SAMPAIO, L. A. et al. Percepção dos enfermeiros da Estratégia Saúde da Família sobre o uso da Fitoterapia. Revista Mineira Enfermagem. jan/mar; 17(1): 76-84,2013.

SANTOS, R.L. et al. Análise sobre a fitoterapia como prática integrativa no Sistema Único de Saúde. Rev. Bras. Plantas Med., v. 13, n. 4, p. 486-91, 2011. https://doi.org/10.1590/ $\underline{\text { S1516-05722011000400014 }}$

SCHIAVO, M. et al. Avaliação do uso de Plantas Medicinais por mulheres residentes em Ijuí/RS. Salão do Conhecimento, v. 2, n. 01, 2014.

SILVA, J. C. da. et al. Relato de experiência sobre o uso de plantas medicinais por uma comunidade do município de Uruguaiana/RS. 
Journal of Nursing and Health, v. 1, n. 2, p. 334-345, 2011.

SILVEIRA, P.F.; BANDEIRA, M.A.M.; ARRAIS, P.S.D. Farmacovigilância e reações adversas às plantas medicinais e fitoterápicos: uma realidade. Revista Brasileira de Farmacognosia, v. 18, n. 4, p. 618-626, 2008. https://doi.org/10.1590/S0102-695X2008000400021 TEIXEIRA, A. H. et al. Conhecimento popular sobre o uso de plantas medicinais no $\mathrm{Mu}-$ nicípio de Sobral-Ceará, Brasil. SANARERevista de Políticas Públicas, v. 13, n. 1, 2014.

VÁSQUEZ, S. P. F; MENDONÇA, M. S.; NODA, S. N. Etnobotânica de plantas medicinais em comunidades ribeirinhas do Município de Manacapuru, Amazonas, Brasil. Instituto Nacional de Pesquisas da Amazônia, v. 44, n.4, p.457 - 472, 2014. https://doi.org/10.1590/1809-4392201400423

VEIGA JÚNIOR, V.F.; PINTO, A.C.; MACIEL, M.A.M. Plantas medicinais: cura segura? Química Nova. São Paulo, v. 28, n. 3, p. 519-528. maio/jun. 2005. https://doi.org/ 10.1590/S0100-40422005000300026

VEIGA JUNIOR, V.F. Estudo do consumo de plantas medicinais na Região Centro-Norte do Estado do Rio de Janeiro: aceitação pelos profissionais de saúde e modo de uso pela população. Revista Brasileira de Farmacognosia, v. 18, n. 2, p. 308-313, 2008. https://doi.org/10.1590/S0102-695X2008000200027

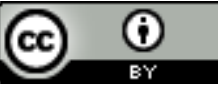

License information: This is an openaccess article distributed under the terms of the Creative Commons Attribution License, which permits unrestricted use, distribution, and reproduction in any medium, provided the original work is properly cited.

Artigo recebido em 22 de agosto de 2018.

Avaliado em 04 de setembro de 2019.

Aceito em 06 de setembro de 2019.

Publicado em 02 de outubro de 2019.

\section{Como citar este artigo (ABNT):}

SOUZA, Mauricio José Cordeiro; LOBATO, Samara Lutyelle Xavier; MENEZES, Rubens Alex de Oliveira. Conhecimento tradicional de plantas medicinais na comunidade ribeirinha do Igarapé Banha no Município de Mazagão - Amapá, Amazônia brasileira. Estação Científica (UNIFAP), Macapá, v. 9, n. 1, p. 51-62, jan./mar. 2019. 